\title{
Bipartite entanglement entropy in fractional quantum Hall states
}

\author{
O.S. Zozulya, ${ }^{1}$ M. Haque ${ }^{2}$ K. Schoutens, ${ }^{1}$ and E. H. Rezayi ${ }^{3}$ \\ ${ }^{1}$ Institute for Theoretical Physics, University of Amsterdam, \\ Valckenierstraat 65, 1018 XE Amsterdam, the Netherlands \\ ${ }^{2}$ Max-Planck Institute for the Physics of Complex Systems, Nöthnitzerstr. 38, 01187 Dresden, Germany \\ ${ }^{3}$ Dept. of Physics, California State University, Los Angeles, California 90032, USA
}

(Dated: May 29, 2007)

\begin{abstract}
We present a detailed analysis of bipartite entanglement entropies in fractional quantum Hall (FQH) states, considering both abelian (Laughlin) and non-abelian (Moore-Read) states. We derive upper bounds for the entanglement between two subsets of the particles making up the state. We also consider the entanglement between spatial regions supporting a FQH state. Using the latter, we show how the so-called topological entanglement entropy $\gamma$ of a FQH state can be extracted from wavefunctions for a limited number of particles.
\end{abstract}

PACS numbers: 03.67.Mn, 71.10.-w, 73.43.-f

\section{INTRODUCTION}

The fractional quantum Hall (FQH) states have long fascinated the condensed-matter community due to their remarkable transport properties and the exotic nature of their quasiparticle excitations. It is in the context of FQH states that the notion of topological order in gapped two-dimensional states first arose. ${ }^{1}$ Recently there has been enhanced interest in FQH states with non-abelian statistics ${ }^{2-4}$ due to the possibility of implementing quantum computation schemes topologically protected from decoherence. ${ }^{5}$ The unusual features of FQH states have been notoriously difficult to characterize using traditional condensed-matter concepts such as local order parameters and $n$-point correlation functions.

In a separate development, recent years have seen growing understanding that entanglement measures borrowed from the discipline of quantum information can be useful in probing global features of quantum manyparticle states. ${ }^{6-9}$ It is thus natural to ask what features of FQH states can be characterized by entanglement measures. In a recent short report, ${ }^{10}$ three of the present authors have shown that one such entanglement measure, the bipartite entanglement entropy, indeed elucidates the subtle correlations and topological order in the simplest FQH states, those in the so-called Laughlin sequence. The bipartite entanglement entropy is defined by partitioning the system under question into two blocks $A$ and $B$, and using the reduced density matrix of one part (e.g., $\rho_{A}=\operatorname{tr}_{B} \rho$ obtained by tracing over $B$ degrees of freedom) to calculate the von Neumann entropy $S_{A}=-\operatorname{tr}\left[\rho_{A} \ln \rho_{A}\right]$.

In Ref. 10, numerical calculations of the entanglement entropy between two spatial regions allowed us to extract from the Laughlin wavefunctions the so-called topological entanglement entropy, a concept introduced in Ref. 11,12. [For brevity we write topological entropy where no confusion can arise.] In addition, we provided results on the entanglement entropy between subsets of the particles making up the state. We showed that such particle entanglements are bounded by expressions that manifest the exclusion statistics in the Laughlin states.

In this article, we present a systematic discussion of bipartite entanglement entropies for FQH states, elaborating on our results in Ref. 10. In addition to the abelian Laughlin (L) states, we consider a series of nonabelian FQH states: the Moore-Read (MR) (or pfaffian) states. ${ }^{2,13,14}$ In planar geometry, the respective wavefunctions are given by

$$
\begin{aligned}
\Psi_{\mathrm{L}}\left(\left\{z_{i}\right\}\right) & =\prod_{i<j}\left(z_{i}-z_{j}\right)^{m} e^{-\sum_{i}\left|z_{i}\right|^{2} / 4} \\
\Psi_{\mathrm{MR}}\left(\left\{z_{i}\right\}\right) & =\operatorname{Pf}\left(\frac{1}{z_{i}-z_{j}}\right) \prod_{i<j}\left(z_{i}-z_{j}\right)^{m} e^{-\sum_{i}\left|z_{i}\right|^{2} / 4},
\end{aligned}
$$

with Pf denoting the antisymmetric Pfaffian symbol. We shall here consider these same states in spherical geometry, so as to eliminate boundary effects.

For both series of states, we derive upper bounds $S_{A}^{\text {bound }}$ for particle entanglement entropies. A marked difference between the $m=3$ Laughlin state and the $m=2$ Moore-Read states is that in the latter the leading correlations have a 3 -body nature, whereas those in the Laughlin states are 2-body effects. This difference is nicely manifested in the leading terms of a $1 / N$ expansion of the upper bounds $S_{A}^{\text {bound }}$, which are given by (see section III below for details)

$$
\begin{aligned}
m= & 3 \text { Laughlin state : } \\
& S_{A}^{\text {bound }}=S_{A}^{F}-\frac{2}{3 N} n_{A}\left(n_{A}-1\right)+\ldots \\
m= & 2 \text { Moore }- \text { Read state : } \\
& S_{A}^{\text {bound }}=S_{A}^{F}-\frac{3}{4 N^{2}} n_{A}\left(n_{A}-1\right)\left(n_{A}-2\right)+\ldots
\end{aligned}
$$

Another marked difference between the abelian and non-abelian states is in the value for the topological entropy $\gamma$. Comparing the Laughlin and Moore-Read states at the same filling fraction $\nu=1 / m$ we have

$$
\gamma_{\mathrm{L}}=\ln \sqrt{m}, \quad \gamma_{\mathrm{MR}}=\ln \sqrt{4 m},
$$


the difference being due to the non-abelian nature of the Moore-Read states (see section IV for details). In this article we extract values for $\gamma$ directly from wavefunctions for a limited number of particles in spherical geometry (up to $N=10$ for the $m=3$ Laughlin state and up to $N=18$ for the $m=2$ Moore-Read state), finding values that are consistent with the expected result. These results illustrate how the entanglement entropy can be used in diagnosing the topological order for a FQH state that is only known in the form of wavefunctions for a limited number of particles, as is often the case in numerical studies.

In Section II we give a general discussion of possible bipartite entanglement measures in itinerant many body systems, carefully distinguishing between particle and spatial partitioning schemes. In Section III we present analytical and numerical results for particle entanglement in the Laughlin and Moore-Read FQH states. In this, the eigenvalue distribution of the reduced density matrix $\rho_{n_{A}}$ plays a central role. In subsection IIIF we relate the eigenvalue distribution for $\rho_{n_{A}=2}$ to the twoparticle correlation function $g_{2}(r)$. In Section IV we discuss spatial partitioning, paying particular attention to the numerical procedure followed in extracting the topological entropy $\gamma$.

While most results in this article are for fermionic FQH states (meaning $m$ odd in the Laughlin sequence and $m$ even for the Moore-Read states), we briefly comment on bosonic states in subsection III C.

\section{PARTITION CHOICES ON THE SPHERE}

The entanglement entropy, being a bipartite measure of entanglement, depends on the particular partitions being considered. Obviously, a many-particle system can be partitioned in many ways. Rather than asking which partition is the "correct" one, we find it more useful to ask what information one can extract from various kinds of partitioning. Accordingly, we have partitioned both the spatial degrees of freedom, and the particles themselves. We find that both schemes are useful, for revealing distinct features of the many-particle state.

The kinds of partitioning one is able to study depend on the available degrees of freedom. Our calculations are all performed for FQH states in a spherical geometry. ${ }^{20,21}$ In this representation the fermions are placed on a sphere containing a magnetic monopole. The magnetic orbitals of the relevant Landau level are then represented as angular momentum orbitals; the total angular momentum is half the number of flux quanta, $L=\frac{1}{2} N_{\phi}$. The $N_{\phi}+1$ orbitals are labeled either $l=0$ to $N_{\phi}$ or $L_{z}=-L$ to $+L$. For $N$ particles at fractional filling $\nu=1 / m$, one finds the interesting FQH states for $N_{\phi}=m N-S$, where $S$ is a finite-size shift. The Laughlin states appear at $S=m$ while for the Moore-Read states $S=m+1$. The "filling" acquires the usual meaning $\nu=N / N_{\phi}$ only in the thermodynamic limit. The orbitals are each localized around a "circle of latitude" on the sphere, with the $l=0$ orbital localized near one "pole."

Since the FQH wavefunctions on a sphere are obtained in terms of orbital occupancies, one can either partition orbitals or partition particles. Because of the spatial arrangement of the orbitals, partitioning orbitals is in fact equivalent to partitioning spatial regions. The difference between spatial and particle partitioning has not been stressed in the literature because the most common systems studied (in the context of entanglement entropies in many-particle states) are spin models, for which there is no such distinction. In the cases of itinerant particles where there is a difference, the common default scheme has been spatial partitioning. In particular, conformal field theory results on entanglement scaling ${ }^{8,15}$ and the distinction between gapless and gapped states observed in entanglement scaling ${ }^{6}$ actually pertain to the blocking of space rather than the particles or spins themselves. The definition of the topological entropy for twodimensional topologically ordered states is also based on the entanglement entropy between spatial blocks. ${ }^{11,12}$

In previous work, ${ }^{10}$ three of the present authors studied the entanglement entropy between subsets of particles making up a Laughlin FQH state. We presented upper bounds and gave an interpretation in terms of exclusion statistics. In this paper we extend these results to the Moore-Read states, where the exclusion effects are more intricate. We refer to Refs. 16-18 for other studies of particle entanglement properties.

For orbital or spatial partitioning, we define block $A$ to be the first $l_{A}$ orbitals, extending spatially from one pole of the sphere out to some latitude. In the thermodynamic limit, this is equivalent to choosing a disk-shaped block $A$ within an infinite planar system. In this limit, since each orbital $l$ is associated with a wavefunction of the form $z^{l} e^{-|z|^{2} / 4}$ in usual complex coordinate language, a disk with $l_{A}$ orbitals has radius $\propto \sqrt{l_{A}}$.

The spatial arrangement of the orbitals constrains us to either disc or ring-shaped spatial regions as the $A$ partition. Since the orbital indexes do not give us access to the full two-dimensional degrees of freedom, it is not possible to experiment with the various kinds of topologically nontrivial partitions suggested by Preskill and Kitaev, ${ }^{11}$ Levin and Wen, ${ }^{12}$ and Furukawa and Misguich. ${ }^{19}$ However, as we have reported previously, ${ }^{10}$ the spherical geometry is sufficient to probe the topological entropy of FQH wavefunctions.

Comparing particle and spatial entanglement entropies, we remark that the effect of correlations is opposite between the two cases. For particle partitioning, the maximal entropy is realized for uncorrelated fermions; correlations tend to lower $S_{A}$ from the fermion bound $S_{A}^{F}$. Spatial entanglement, on the other hand, is entirely due to correlations. The point is illustrated by considering the $m=1$ Laughlin state, where the fermions are uncorrelated. Here the particle entanglement entropy equals $S_{A}^{F}$, while the spatial entanglement entropy vanishes. 


\section{ENTANGLEMENT FOR PARTICLE PARTITIONING}

In this section we provide close upper bounds to the entropy of entanglement between $n_{A}$ particles of the state and the remaining $n_{B}=N-n_{A}$ particles. We also discuss the $n_{A}$-particle reduced density matrices $\rho_{n_{A}}$ that arise in this context.

\section{A. Multiplet structure and particle entropy bounds}

For FQH states on a sphere, the $n_{A}$-particle reduced density matrices $\rho_{n_{A}}$ commute with the total angular momentum operators $\mathbf{L}_{n_{A}}^{2}$ and $L_{n_{A}}^{z}$ of the selected $n_{A}$ particles. This implies that the eigenvalues of $\rho_{n_{A}}$ are organized in a multiplet structure of the corresponding $S U(2)$ algebra: an eigenvalue for total angular momentum $L_{n_{A}}$ will be $\left(2 L_{n_{A}}+1\right)$-fold degenerate.

For $n_{A}=2$ fermions, each having angular momentum $L$, the 2-particle states have total angular momenta $L_{2}=$ $2 L-1,2 L-3, \ldots, 1(0)$, for $L$ integer (half-integer), giving a total number of $(2 L+1)(2 L) / 2$ states. A naive upper bound to the entanglement entropy is thus

$$
S_{n_{A}=2} \leq \ln [(2 L+1)(2 L) / 2] .
$$

Inspecting the explicit structure of the fermionic Laughlin states with $m=3,5, \ldots$, one finds that the eigenvalues corresponding to 2-particle states with $L_{2}=2 L-1$, $2 L-3, \ldots, 2 L-(m-2)$ all vanish. The reason is that the correlations in the Laughlin states are such that particles cannot come too close together. For example, if a first fermion occupies the $l=0$ orbital, localized near the north pole, the Laughlin wavefunction has zero amplitude for finding a second fermion in orbitals $l=1$, $l=2, \ldots, l=m-1$. The highest possible value of the angular momentum of the two fermions combined is thus $L_{2}=L+(L-m)$. The remaining number of non-zero eigenvalues is $(2 L+(2-m))(2 L+(1-m)) / 2$, leading to an improved bound on the entropy $S_{n_{A}=2}$

$$
S_{n_{A}=2} \leq \ln [(2 L+(2-m))(2 L+(1-m)) / 2]
$$

with $2 L=m(N-1)$ as before. For $n_{A}>2$, the multiplet structures are more complicated and we need to resort to a different method for finding a non-trivial upper bound to the particle entropy. In the next subsection we give a general derivation for both the Laughlin and the MooreRead series of fermionic FQH states.

\section{B. Upper bounds for fermionic states}

For $N$ fermionic particles, $n_{A}$ particles in the $A$ block, and the total number of orbitals given by $N_{\phi}+1=2 L+1$, fermionic statistics lead to an obvious upper limit $S_{A}^{\mathrm{F}}$ to the entropy $S_{A}$

$$
S_{A} \leq S_{A}^{\mathrm{F}}=\ln \left(\begin{array}{c}
N_{\phi}+1 \\
n_{A}
\end{array}\right)
$$

In the FQH states the correlations are such that the particles avoid each other and the entropy is further reduced. To obtain a handle on this, one may reason as follows. The model FQH states in the Laughlin and Moore-Read series can be characterized as zero-energy eigenstates of a Hamiltonian penalizing pairs and/or triplets of particles coming to the same position. After tracing out the coordinates for the $B$ set, the dependence on those in the $A$ set is such that one still has a zero-energy eigenstate. However, the number of orbitals available to the $A$ particles is larger than what is needed to make the model FQH state in the $A$ sector, and one instead has a certain number of quasi-holes on top of the $A$ set model state. The total ground state degeneracy for this situation has been studied in the literature: see Ref. 13 for the Laughlin and Moore-Read states and Ref. 22 for the Read-Rezayi and Ardonne-Schoutens series of non-abelian FQH states.

For the Laughlin states the details are as follows. The $N$-particle Laughlin state is realized on a total of $N_{\phi}+1$ Landau orbitals, corresponding to $N_{\phi}=m(N-1)$ flux quanta. The Laughlin state for $n_{A}$ particles would need $N_{\phi}^{A}=m\left(n_{A}-1\right)$ flux quanta; we thus have an excess flux of $\Delta N_{\phi}=N_{\phi}-N_{\phi}^{A}=m\left(N-n_{A}\right)$. With the Laughlin gauge argument this corresponds to the presence of $n_{\mathrm{qh}}=\Delta N_{\phi}$ quasi-holes over the groundstate. According to Ref. 13 each of the quasi-holes has a number of $n_{A}+1$ effective orbitals to choose from, with bosonic counting rules (meaning that two or more quasi-holes can be in the same effective orbital). This gives a number of quasi-hole states equal to

$$
\left(\begin{array}{c}
\left(n_{A}+1\right)+n_{\mathrm{qh}}-1 \\
n_{\mathrm{qh}}
\end{array}\right),
$$

leading to the following upper bound to the entropy $S_{A}$

$$
S_{A}^{\text {bound }}=\ln \left(\begin{array}{c}
N_{\phi}+1-(m-1)\left(n_{A}-1\right) \\
n_{A}
\end{array}\right) .
$$

We remark that this expression has a clear interpretation in terms of exclusion statistics: the counting factor in Eq. (4) gives the number of ways $n_{A}$ particles can be placed in $N_{\phi}+1$ orbitals, in such a way that a particle placed in a given orbital $l$ excludes particles from orbitals $l^{\prime}$ with $\left|l-l^{\prime}\right|<m$.

In a $1 / N$ expansion we find (assuming $n_{A} \ll N$ )

$$
\begin{aligned}
& S_{A}^{\mathrm{F}}-S_{A}^{\text {bound }}= \\
& \quad \frac{1}{N} \frac{m-1}{m} n_{A}\left(n_{A}-1\right) \\
& \quad+\frac{1}{N^{2}} \frac{m-1}{2 m^{2}} n_{A}\left(n_{A}-1\right)\left[2 m+\left(n_{A}-1\right)\left(m+n_{A}-4\right)\right] \\
& \quad+\mathcal{O}\left(1 / N^{3}\right)
\end{aligned}
$$

The particle entropy reaches a maximum for $n_{A}=$ $N / 2$. For this case our Eq. (4) gives, in the limit of large $N$,

$$
S_{n_{A}=N / 2} \leq N[(m+1) \ln (m+1)-m \ln (m)] / 2 .
$$


This bound is sharper than a bound recently presented in Ref. 17, which gives a larger coefficient for the linearin- $N$ behavior.

For the fermionic Moore-Read states at $\nu=1 / m$, with $m=2,4, \ldots$, we can reason in a similar way, with now $N_{\phi}=m(N-1)-1$. As for the Laughlin states we have an excess flux of $\Delta N_{\phi}=N_{\phi}-N_{\phi}^{A}=m\left(N-n_{A}\right)$ but now the number of quasi-holes is twice this number due to the fact that the fundamental quasi-holes correspond to half a flux quantum. Thus, $n_{\mathrm{qh}}=2 \Delta N_{\phi}$. We now take from Ref. 13 the following result for the total quasi-hole degeneracy

$$
\sum_{F \equiv n_{A}}^{n_{A}}\left(\begin{array}{c}
n_{\mathrm{qh}} / 2 \\
F
\end{array}\right)\left(\begin{array}{c}
\left(n_{A}-F\right) / 2+n_{\mathrm{qh}} \\
n_{\mathrm{qh}}
\end{array}\right) .
$$

This gives us an upper bound $S_{A}^{\text {bound }}$ as before.

Putting $m=2$, one easily checks that $S_{A}^{\text {bound }}$ coincides with $S_{A}^{\mathrm{F}}$ for $n_{A}=2$. In a $1 / N$ expansion, the leading deviation from $S_{A}^{F}$ is a 3 -body term at order $1 / N^{2}$,

$$
S_{A}^{\mathrm{F}}-S_{A}^{\text {bound }}=\frac{1}{N^{2}} \frac{3}{4} n_{A}\left(n_{A}-1\right)\left(n_{A}-2\right)+\ldots
$$

This result nicely illustrates the fact that the leading correlations in the $m=2$ Moore-Read state have a 3-body character: the wave-function vanishes if at least three particles come to the same position.

For $m \neq 2$ the leading correlations do have a 2-body character, as for the Laughlin states,

$$
S_{A}^{\mathrm{F}}-S_{A}^{\mathrm{bound}}=\frac{1}{N} \frac{m-2}{m} n_{A}\left(n_{A}-1\right)+\ldots
$$

Inspecting the particle entanglement at $n_{A}=N / 2$ and for $N$ large, our bound implies that for the $m=2$ MooreRead state

$$
S_{n_{A}=N / 2} \leq 1.044 N
$$

This bound is reduced from the Fermi bound $S_{A}^{F} \sim$ $N(4 \ln 4-3 \ln 3) / 2 \sim 1.125 N$, but it is larger than the bound for the $m=2$ (bosonic) Laughlin state, which has asymptotic form $N(3 \ln 3-2 \ln 2) / 2 \sim .955 N$. This indicates that, at equal filling $\nu=1 / 2$, the particles in a Moore-Read state are more entangled than those in a Laughlin state.

The quasi-hole counting rules for the order- $k$ clustered spin-polarized (Read-Rezayi) and spin-singlet (ArdonneSchoutens) states are all known in the literature. ${ }^{22}$ They can be used to generalize the upper bounds on particle entanglement entropy given in this subsection to these more intricate non-abelian FQH states.

\section{Bosonic quantum Hall states}

We briefly comment on the case of bosonic FQH states. The realization that a rapidly rotating Bose gas may eventually enter a regime of bosonic quantum Hall states motivates the theoretical study of the effects of bosonic statistics.

We consider bosonic Laughlin states at filling fraction $\nu=\frac{1}{m}$ with $m=2,4, \ldots$. The naive upper bound to the the entropy associated to placing $n_{A}$ bosons in $N_{\phi}+1$ orbitals is

$$
S_{A}^{\mathrm{B}}=\ln \left(\begin{array}{c}
N_{\phi}+n_{A} \\
n_{A}
\end{array}\right)
$$

The expression for $S_{A}^{\text {bound }}$ remains unchanged, giving the following leading correction in a $1 / N$ expansion

$$
S_{A}^{\mathrm{B}}-S_{A}^{\mathrm{bound}}=\frac{1}{N} n_{A}\left(n_{A}-1\right)+\ldots
$$

For a bosonic Moore-Read state, with filling fraction $\nu=1 / m$ with $m=1,3, \ldots$, the leading $1 / N$ correction becomes

$$
S_{A}^{\mathrm{B}}-S_{A}^{\text {bound }}=\frac{1}{N} \frac{m-1}{m} n_{A}\left(n_{A}-1\right)+\ldots
$$

In the case $m=1$ the leading correlations have 3-body character, leading to the vanishing of the leading $1 / N$ correction.

\section{Numerical results}

In deriving the upper bound $S_{A}^{\text {bound }}$ we relied on the fact that a certain number of eigenvalues of the reduced density matrix vanish. The bounds would be exact if all non-zero eigenvalues were equal, but since they are not the bounds overestimate the actual values for the entropies.

Fig. 1 plots the eigenvalues for the $n_{A}=2$-particle reduced density matrix for $N=9$ particles on a sphere in the $m=3$ Laughlin state, for which the single particle angular momentum is $L=12$. The horizontal axis represents the degeneracy $2 L_{2}+1$ of the eigenvalues, in descending order. The eigenvalue at $L_{2}=2 L-1=23$, with degeneracy 47 , vanishes; the non-zero eigenvalues show some scatter around an asymptotic value. Due to this scatter the entropy $S=5.509$ is somewhat lower than the upper bound $S_{A}^{\text {bound }}=5.533$.

An important difference between the $m=3$ Laughlin and the $m=2$ Moore-Read states is the absence of vanishing eigenvalues for the 2-particle reduced density matrix. The eigenvalue distribution shown in Fig. 2 illustrates this point.

In the $m=2$ Moore-Read state, there are vanishing eigenvalues in the reduced density matrix of $n_{A} \geq 3$ particles. The number of nonzero eigenvalues predicted by Eq. (7) agrees with numerical results. For example, for $n_{A}=3$ and $N=10$ particles there are 770 nonvanishing eigenvalues, in agreement with Eq. (7).

In Figs. 3, 4 we compare numerically computed particle entanglement entropies with the bounds derived above. 


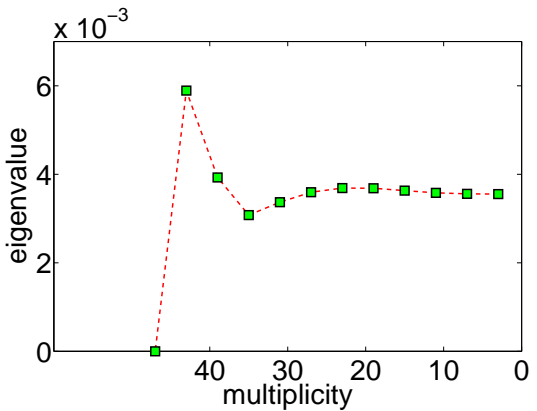

FIG. 1: (Color online) Eigenvalues for the 2-particle reduced density matrix, plotted against their multiplicities, for $N=9$ particles in the $m=3$ Laughlin state.

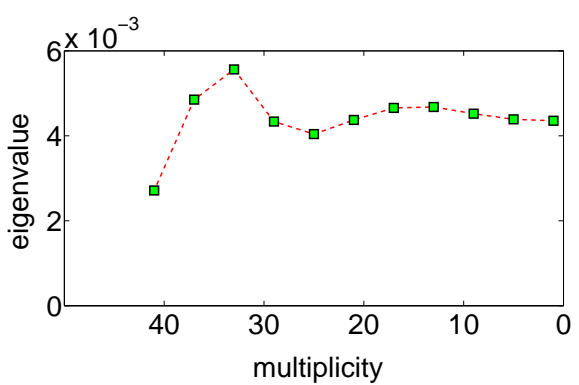

FIG. 2: (Color online) Eigenvalues for the 2-particle reduced density matrix, plotted against their multiplicities, for $N=12$ particles in the $m=2$ Moore-Read state.
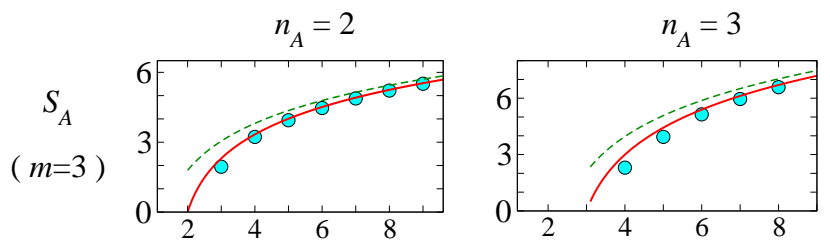

No. of particles in wavefunction, $N$

FIG. 3: (Color online) Entanglement entropy for $n_{A}=2$ and $n_{A}=3$ particles for the $m=3$ Laughlin state. Dots are numerical exact values, the dotted line represents $S_{A}^{F}$ and the solid curve is the bound $S_{A}^{\text {bound }}$.
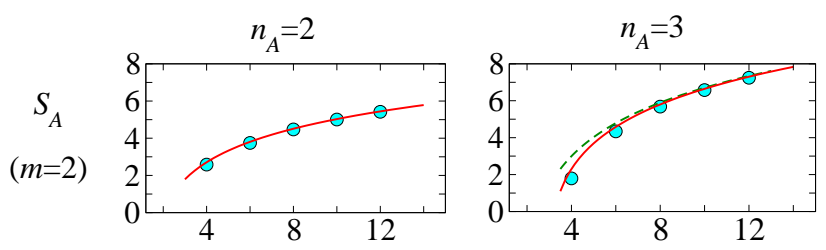

No. of particles in wavefunction, $N$

FIG. 4: (Color online) Entanglement entropy for $n_{A}=2$ and $n_{A}=3$ particles for the $m=2$ Moore-Read state. Dots are numerical exact values, the dotted line represents $S_{A}^{F}$ and the solid curve is the bound $S_{A}^{\text {bound }}$.

\section{E. Corrections to $S_{A}^{\text {bound }}$ due to eigenvalue spread}

It is interesting to consider in some detail the deviation between the bounds $S_{A}^{\text {bound }}$ and the actual entropies computed numerically. As mentioned above, this deviation arises from the fact that the non-zero eigenvalues of the reduced density matrices are not all equal.

To estimate the effect on $S_{n_{A}}[N]$ of the spread in the non-zero eigenvalues, we do a rough modeling of the eigenvalue distribution (Fig. 1) of $\rho_{n_{A}=2}$ for the $m=3$ Laughlin state. For this case the number $D$ of non-zero eigenvalues is $D=(3 N-4)(3 N-5) / 2$. If these nonzero eigenvalues were all equal (to $1 / D$ ), the entanglement entropy would have the maximum value $S_{D}=\ln D$, which is the predicted upper bound (4). We now take into account the deviations from $1 / D$, guided by Fig. 1, with the following toy distribution: we take $D_{0}$ out of $D$ of the eigenvalues to be equal to $\alpha / D$, with $\alpha>1$, while the rest of the eigenvalues are at value $\beta / D, \beta<1$, such that the sum of eigenvalues is unity. Assuming $D_{0} / D \ll 1$ leads to

$$
S \approx \ln D-(\alpha \ln \alpha-\alpha+1) \frac{D_{0}}{D} .
$$

Guided by the eigenvalue distributions in Fig. 1, we assume that $D_{0} / D$ is of order $1 / N$; for concreteness we put $D_{0}$ equal to the multiplicity of the largest eigenvalue, which is $6 N-7$. Taking $\alpha$ between 1.2 and 1.5 (as observed for the largest available Laughlin wavefunctions) gives a $1 / N$ correction in the entropy with coefficient in the range $0.03-0.14$.

Fitting the difference $S_{A}^{F}-S_{n_{A}=2}$ to a form $a / N+b / N^{2}$ gives a coefficient $a \simeq 1.38$. The vanishing eigenvalues account for $a=4 / 3$, see Eq. (5), and we see that the remaining difference $\delta a \sim 0.05$ is consistent with the $1 / N$ correction due to the spread in the non-zero eigenvalues.

We made similar estimates for a the $m=2$ MooreRead state with up to $N=12$ particles, where the eigenvalues are all non-zero and $S_{A}^{\text {bound }}$ agrees with $S_{A}^{F}$. In this case the deviation between data and bound show a $1 / N$ dependence with a coefficient of about 0.14 .

These considerations are of some general interest, as they make the point that a $1 / N$ expansion of particle entanglement entropies are indicative of correlations in a many-body state. In the concrete case studied here, the sizeable value of the leading $1 / N$ correction in the Laughlin state indicates strong 2-body correlations, while the small value for the Moore-Read state indicates the absence of such correlations.

\section{F. Reduced density matrices and correlation functions}

Since the $n_{A}$-particle reduced density matrices $\rho_{n_{A}}$ are obtained by integrating out all but $n_{A}$ of the particles, one expects these matrices to be related to the $n_{A}$-particle correlation functions. In this subsection we 


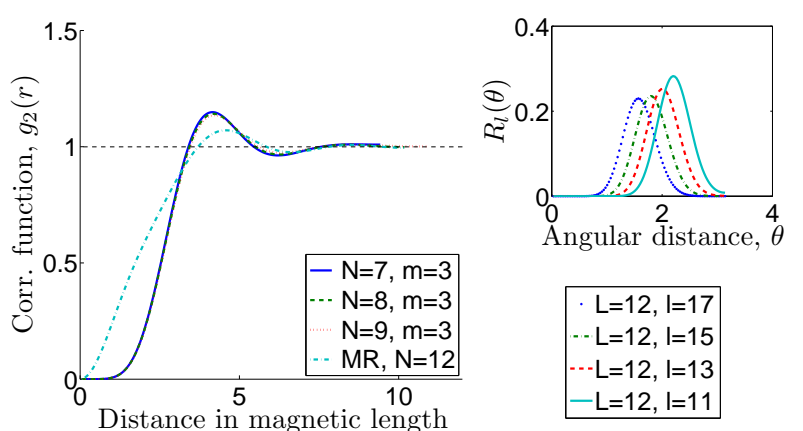

FIG. 5: (Color online) Two-particle correlation as a function of distance in units of the magnetic length, for the $m=3$ Laughlin state with $N=7,8,9$ particles and for the $m=2$ Moore-Read state with $N=12$.

study this relation for the case $n_{A}=2$. In particular, the eigenvalue distributions of $\rho_{n_{A}=2}$ in Figs. 1, 2, although discrete, are reminiscent of the well-known two-particle correlation functions $g_{2}(r)$ for Laughlin and Moore-Read states. We will show that the eigenvalue distributions are in fact very closely related to the correlation functions - the eigenvalue distribution function is a kind of discretized version of $g_{2}(r)$.

The two-particle correlation function $g_{2}(r)$ is conventionally defined as

$$
\begin{aligned}
& g_{2}(r)=\frac{N(N-1)}{n^{2}} \times \\
& \frac{\int d^{2} r_{3} \cdots d^{2} r_{n} \Psi^{*}\left(0, r, r_{3}, \cdots, r_{n}\right) \Psi\left(0, r, r_{3}, \cdots, r_{n}\right)}{\langle\Psi \mid \Psi\rangle}
\end{aligned}
$$

where $N$ is the number of particles and $n$ is a density, which is chosen such that $g_{2}(r) \stackrel{r \rightarrow \infty}{=} 1$.

We express the 2-particle reduced density matrix $\rho_{2}$ on a sphere in a basis of polar spherical coordinates. Because of the rotational symmetry it should be a function of the angular distance $\theta$ between the two particles. In Appendix A we show that $\rho_{2}(\theta)$ can be written in the form

$$
\rho_{2}(\theta)=\sum_{l} \lambda_{l} \frac{2 l+1}{4 \pi} R_{l}(\theta) .
$$

In this expression, $\lambda_{l}$ is the eigenvalue with multiplicity $2 l+1$, corresponding to the total angular momentum of the two particles equal to $l$. The functions $R_{l}(\theta)$ are explicitly given in Eq. (A12).

Since the distance $r$ between two particles is simply equal to $r=R \theta$, with $R$ the radius of the sphere, the 2particle correlation function $g_{2}(r)$ is directly proportional to $\rho_{2}(\theta)$. Through Eq. (16) it is expressed as a transfrom from $l$ space to $\theta$ space, with basis functions $R_{l}(\theta)$. In Fig. 5, we show some curves for $\rho_{2}(\theta)$; they agree with known results.
As illustrated in the inset to Fig. 5, the basis functions $R_{l}(\theta)$ have a peak structure, with the position of the peak depending on the total angular momentum $l$. Large values of $l$ correspond to small angular distances and vice versa. This is easy to understand from the following classical picture. When two particles with angular momenta $L$ have total angular momentum $2 L$, the corresponding vectors $\vec{L}_{1}$ and $\vec{L}_{2}$ should point a the same direction. The angular momentum vector points at the position of the particle on a sphere, therefore two particle should be close to each other. On the other hand, if total angular momentum is zero then the angular momentum vectors should point into opposite directions. This means that particles are placed at the opposite sides of the sphere.

The fact that the $R_{l}(\theta)$ are localized functions, peaked at $\theta$ values monotonically decreasing with $l$, indicates that $g_{2}(r)$ curve is simply a continuous form of the $\lambda_{l}$ versus descending- $(2 l+1)$ curves of Figs. 1 and 2 . The similarity between the discrete $\lambda_{l}$ and the continuous $g_{2}(r)$ curves is not accidental.

At small distances $R_{l}(\theta) \propto\left(\theta^{2}\right)^{2 L-l}$. For a Laughlin state the lowest value of $2 L-l$ is $m$, thus $\rho_{2}(\theta) \propto \theta^{2 m}$. This behavior is a direct consequence of the vanishing of eigenvalues with the largest multiplicities. For the $m=2$ Moore-Read state the lowest value of $2 L-l$ is 1 because there are no vanishing eigenvalues. Therefore at small distances $\rho_{2}(\theta) \propto \theta^{2}$.

Of course, our observations on the 2-particle correlations agree with known results; our main point has been to stress the intimate relation with the eigenvalue distribution of the 2-particle reduced density matrices.

\section{SPATIAL ENTANGLEMENT AND TOPOLOGICAL ENTROPY}

We now turn to dividing the Landau level orbitals into two blocks and calculating the entropy of entanglement between them. In a previous publication, ${ }^{10}$ we used this scheme to extract the topological entropy of the Laughlin state. Here, we mainly focus on the $(m=2)$ Moore-Read state. After reviewing the topological entropy $\gamma$ and the total quantum dimension $\mathcal{D}$, especially in the context of the Moore-Read state (IV A), we detail some issues with taking the thermodynamic limit necessary for extracting $\gamma$ from numerical data (IVB), and then present our numerical results (IV C). We also present observations on the spectral structure of the reduced density matrices (IV D).

\section{A. Topological entropy for the Moore-Read state}

For spatial partitioning of many-particle states, the general rule ("area law") is that the entanglement entropy scales as the size of the boundary between the $A$ and $B$ blocks. ${ }^{23}$ Subtle information about the nature 
of the many-particle state can be provided by the presence or absence of logarithmic corrections, values of coefficients, or subleading terms in this basic relationship. For topologically ordered states in two dimensions, the following theorem has been presented recently ${ }^{11,12}$ concerning the scaling of entanglement entropy between spatial partitions. If $L$ is the length of the boundary between the two blocks, the entanglement entropy scales as $S_{A}=\alpha L-\gamma+\mathcal{O}\left(L^{-1}\right)$. As usual the scaling law applies to situations where $A$ is large and the total system is infinite. The subleading term $\gamma$ is called the topological entanglement entropy. The striking result of Refs. 11,12 has been that it can be expressed as the logarithm of a quantity $\mathcal{D}$ known as the total quantum dimension of the topological field theory describing the topological order of the state. The total quantum dimension is given by

$$
\mathcal{D}=\sqrt{\sum_{i} d_{i}^{2}}
$$

where the $d_{i}$ 's are the quantum dimensions of the individual sectors making up the topological field theory. These quantum dimensions are set by fusion rules of the fundamental anyons in the field theory.

The topological field theory for a $\nu=1 / m$ Laughlin state has a fundamental anyon (of fractional charge $q=$ $e / m)$, which generates $m$ abelian sectors. The quantum dimension $d_{i}$ is unity in all sectors so that $\mathcal{D}=\sqrt{m}$ for the $\nu=1 / m$ Laughlin state. For $m=3$ this gives $\gamma=\ln \sqrt{3} \simeq 0.55$.

For states with non-abelian quasiparticles, the situation is more interesting because some anyon sectors contribute $d_{i}>1$. Details for some examples have been provided in Refs. 11,24. In particular, for the $m=2$ Moore-Read state, there are six sectors (two each of quasiparticles denoted by $I, \sigma, \psi)$ which contribute $d_{I}=1, d_{\sigma}=\sqrt{2}, d_{\psi}=1$, leading to $\mathcal{D}=\sqrt{8}$ and $\gamma \simeq 1.04$. The non-abelian nature shows up in the fact that $\gamma$ is larger than $\ln \sqrt{6}$, six being the degeneracy of the $m=2$ Moore-Read state on the torus.

A compact general expression for the total quantum dimension for a Read-Rezayi state with order- $k$ clustering and at filling fraction $\nu=k /(k M+2)$ is (see also Ref. 24)

$$
\mathcal{D}_{\mathrm{RR}}[k, M]=\frac{\sqrt{(k+2)(k M+2)}}{2 \sin (\pi /(k+2))} .
$$

It includes the Laughlin states $(k=1, M=m-2)$ and the Moore-Read states $(k=2, M=m-1)$ as special cases. For a general spin-singlet non-abelian FQH state with order $k$ clustering and filling fraction $\nu=2 k /(2 k M+3)$, the result is

$$
\mathcal{D}_{\mathrm{AS}}[k, M]=\frac{(k+3) \sqrt{(2 k M+3)}}{16 \cos (\pi /(k+3)) \sin ^{3}(\pi /(k+3))},
$$

giving $\gamma \simeq 1.62$ for the paired spin-singlet state $(k=2$, $M=1$ ) at $\nu=4 / 7$.
In general, the $M$-dependence of these expressions for total quantum dimensions is linked to the ground state degeneracy in torus geometry. Denoting the latter by $\#[k, M]$ we have the relation

$$
\mathcal{D}[k, M]=\mathcal{D}[k, 0] \sqrt{\frac{\#[k, M]}{\#[k, 0]}},
$$

The conformal field theories underlying the states at $M=0$ are of Wess-Zumino-Witten type $\left(S U(2)_{k}\right.$ for the RR states and $S U(3)_{k}$ for the AS series) and the quantities $\mathcal{D}[k, 0]$ can be expressed in the modular $S$-matrix for these WZW models.

\section{B. $\gamma$ from sphere calculations: extrapolations}

First of all, we note that, since our degrees of freedom are ordered essentially one-dimensionally, we cannot use one of the two-dimensional schemes proposed previously ${ }^{11,12,19}$ in which an appropriate addition/subtraction of the entanglement entropies of several regions cancels the boundary parts of the entropy $\left(S_{A} \rightarrow \alpha L-\gamma\right)$ leaving the subleading term $\gamma$. With the orbital degrees of freedom on a sphere, we can choose only regions corresponding to disks, concentric rings, and combinations thereof. Any combination of entropies of disk- and ring-like regions that cancels out the boundary terms also unfortunately cancels out the $\gamma$ term.

We are thus led to using directly the scaling law, $S_{A} \stackrel{L \rightarrow \infty}{\longrightarrow} \alpha L-\gamma$. Our choice of block $A$ as the first $l_{A}$ orbitals, extending spatially from one pole out to some latitude, corresponds to a disk-shaped block only in the thermodynamic limit. The block area is proportional to the square of $\sqrt{l_{A}}$ while its boundary is proportional to $\sqrt{l_{A}\left(N_{\phi}+1-l_{A}\right)}$; these are equivalent only in the same $N \rightarrow \infty$ limit. One way to numerically access the thermodynamic limit is to take the entanglement entropy of $l_{A}$ orbitals with the rest, for accessible wavefunctions of various sizes $N$, and then take the $N \rightarrow \infty$ limit. The $S_{l_{A}}(N \rightarrow \infty)$ versus $\sqrt{l_{A}}$ points thus obtained should then follow a linear curve at large $l_{A}$, whose vertical intercept gives the topological entropy. Results following this procedure were provided for the $\nu=1 / 3$ Laughlin state in our earlier paper; ${ }^{10}$ here we will focus on the Moore-Read state. The extrapolation of $S_{l_{A}}(N)$ values to the thermodynamic limit is a tricky issue. We therefore discuss the extrapolation in some detail here, providing some general results.

We are interested in the function $S_{l_{A}}(x)$, where $x=$ $1 / N$. We have access to $S_{l_{A}}\left(x_{i}\right)$ at several integer values of $N$, and would like to estimate $S_{l_{A}}(0)$. For each dataset that we have access to (each $l_{A}$; both Laughlin and Moore-Read), we note the following: the $S_{l_{A}}\left(x_{i}\right)$ versus $x_{i}$ values form a monotonic curve and this curve gets flatter (slope magnitude decreases) with decreasing $x$. Two examples can be seen in the inset to Fig. 6 . In other words, the first and second derivatives of the 


\begin{tabular}{llll}
\hline \hline$\omega=2.0$ & & & \\
\hline 3.46601 & 5.21183 & 4.69023 & 4.77647 \\
3.80408 & 4.92355 & 4.74756 & \\
4.01821 & 4.84476 & & \\
4.16743 & & & \\
\hline \hline$\omega=3.0002$ & & & \\
\hline 3.46601 & 4.56058 & 4.51976 & 4.55722 \\
3.80408 & 4.53643 & 4.59865 & \\
4.01821 & 4.56761 & & \\
4.16743 & & & \\
\hline \hline$\omega=2.5926$ & & & \\
\hline 3.46601 & 4.74638 & 4.58027 & \\
3.80408 & 4.65109 & 4.65108 & \\
4.01821 & 4.65108 & & \\
4.16743 & & & \\
\hline \hline
\end{tabular}

TABLE I: Extrapolation using the BST algorithm, using three different $\omega$ values for the same initial dataset (first column), corresponding to $S_{l_{A}=11}(N)$ values for $N=12,14,16$, 18. In the last case, $\omega$ has been tuned to ensure that the sets obtained after first and second iterations converge to the same value, i.e., the lowest elements of the second and third columns are the same.

$S_{l_{A}}(x)$ function have the same sign and neither derivative changes sign.

Motivated by the above observations, we provide the following result. Assuming only that the signs of the first two derivatives of the $S_{l_{A}}(x)$ function are the same and that the signs remain unchanged until $x=0$, we have:

1. The value $S_{0}=S_{l_{A}}\left(x_{0}\right)$ corresponding to the smallest value $x_{0}$ of the available $x_{i}$ is a strict lower (upper) bound for $S_{l_{A}}(0)$ if the $S_{l_{A}}^{\prime}(x)$ is negative (positive).

2. The intercept found by connecting the $S_{l_{A}}(x)$ corresponding to the smallest two $x_{i}$ values $\left(x_{0}, x_{1}\right)$, namely

$$
S_{1}=S_{l_{A}}\left(x_{0}\right)\left(1-\frac{x_{0}}{x_{1}-x_{0}}\right)+S_{l_{A}}\left(x_{1}\right)\left(\frac{x_{0}}{x_{1}-x_{0}}\right),
$$

is a strict upper (lower) bound if $S_{l_{A}}^{\prime}(x)$ is negative (positive) and $S_{l_{A}}^{\prime \prime}(x)$ is positive (negative).

The limits $S_{0}$ and $S_{1}$ thus obtained give us conservative bounds for the required entanglement entropies in the thermodynamic limit, $S_{l_{A}}(N \rightarrow \infty)$. To obtain a sharper extrapolation, one can use various polynomial extrapolations and take the average, as done in our earlier work. ${ }^{10}$ Here, we improve the extrapolation by using the extrapolation algorithm of Bulirsh and Stoer (BST algorithm), based on rational polynomial fraction approximations. ${ }^{25,26}$

The BST procedure involves successive transformations of the original dataset, leading to transformed datasets successively smaller by one element. If the free parameter $(\omega)$ of the algorithm is chosen properly, the successive sets will be more and more convergent and will eventually converge to the $x=0$ value. The parameter $\omega$ is chosen to optimize convergence; how precisely this is done is a non-trivial issue, especially for large datasets.

In the present case where the initial dataset consists of bipartite entanglement entropies, it is particularly desirable to be able to use a small number of initial $S_{l_{A}}(N)$ values, corresponding to the largest available $N$. This is because the symmetry $S_{l_{A}}=S_{N_{\phi}+1-l_{A}}$ makes the $S_{l_{A}}(N)$ data meaningless for $N \rightarrow \infty$ extrapolation when $l_{A}$ gets close to $N_{\phi} / 2=\frac{1}{2}(m N-S)$. In other words, the $S_{l_{A}}(N)$ values used should not be too close to the peak at the midpoint of the respective $S_{l_{A}}(N)$ versus $l_{A}$ curve (e.g., Figure 1 of Ref. 10). This restricts us to the largest few $N$ values.

In Table I, we demonstrate some possible choices of $\omega$ starting from four $S_{l_{A}}(N)$ values. The top example uses $\omega=2$, which is a common choice used for extrapolationbased numerical integration algorithms. In the second example, $\omega$ is tuned to give the "most converged" set after the first iteration, by choosing $\omega$ to minimize the standard deviation of the second column. We find that this procedure does not always lead to great stability for the subsequent iteration results.

In the last example, we have tuned $\omega$ to give the same last value for the sequences obtained after the first and the second iterations. This also guarantees the third (final) iteration to give the same value (Table I), which indicates that the convergence is very good. This procedure has the added advantage that actually only the last three points of the initial dataset are used to determine the values tuned to be equal. We thus get a nicely converged estimate based only on three initial points, which is highly desirable as explained above. This is therefore our method of choice for tuning the free parameter $\omega$ in applying the BST algorithm for our problem.

As a side note, we remark that if the largest available entanglement entropies are not known exactly (e.g., if they are calculated from approximate wavefunctions, or using the DMRG technique), it might be more prudent to use a prescription for choosing $\omega$ that actually uses more than the minimal (three) numbers that we have used here. In the cases reported here and in our earlier work, ${ }^{10}$ we have entanglement entropies calculated from numerically exact wavefunctions, so we have no precision issues to worry about when choosing the number of $S_{l_{A}}(N)$ values to use for the $N \rightarrow$ extrapolation.

Once the extrapolation value is (uniquely) determined by the algorithm outlined above, we need an estimate of the uncertainty. We can use the bounds $\left(S_{0}, S_{1}\right)$ derived earlier to obtain a conservative error estimate:

$$
\min \left(\left|S_{1}-S_{\mathrm{BST}}\right|,\left|S_{\mathrm{BST}}-S_{0}\right|\right)
$$

where $S_{\mathrm{BST}}$ is the extrapolated value obtained by the BST algorithm. 


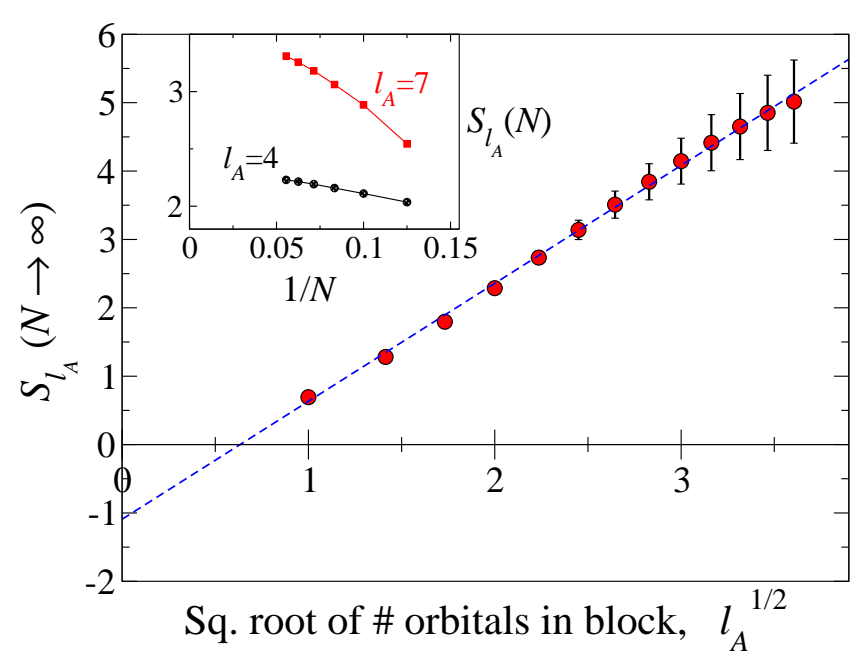

FIG. 6: (Color online.) Entanglement entropies in MooreRead state wavefunctions, extrapolated to the thermodynamic limit. Dashed line is a fit to $-\gamma+c_{1} \sqrt{l_{A}}$, with some points dropped. Inset plots $S_{l_{A}}$ against $1 / N$ for various fixed $l_{A}$.

\section{Numerical results}

Moore-Read state. Fig. 6 shows results of numerical calculations for the $\nu=1 / 2$ Moore-Read state. We used exact wavefunctions up to $N=18$ particles. These wavefunctions were obtained by diagonalizing $\hat{L}^{2}$ in an $L_{z}=0$ Hilbert space spanned by the "squeezed states" 27 . After numerically obtaining the entanglement entropies $S_{l_{A}}(N)$ from these wavefunctions, we obtain estimates and uncertainties for the $N \rightarrow \infty$ extrapolations by the procedure outlined in the previous subsection. The resulting data are plotted in Fig. 6.

The linear $S_{l_{A}}$ versus $\sqrt{l_{A}}$ behavior is expected only for large $l_{A}$; however our large- $l_{A}$ points have the greatest uncertainty. For estimating the topological entropy, we therefore make linear fits after discarding 0 to 5 of the smallest- $l_{A}$ points and/or 0 to 2 of the largest- $l_{A}$ points. This results in estimates of $\gamma$ (magnitude of the vertical intercept) scattered between 0.85 and 1.35 . The error propagated into our $\gamma$ estimate from our extrapolation uncertainties is $\sim 0.3$, larger than that obtained from this scatter. With all this we arrive at the result $\gamma \simeq 1.1 \pm 0.3$, quite consistent with the expected value of $\gamma \simeq 1.04$.

Laughlin state. We used the well-defined procedure of the previous subsection to revisit our previous estimate of the topological entropy for the $\nu=1 / 3$ Laughlin state. ${ }^{10}$ To get the extrapolated $S_{l_{A}}$, we now use the BST estimates rather than doing several polynomial fits. Dropping 0 to 4 of the smallest- $l_{A}$ points and 0 to 2 of the lrgest- $l_{A}$ points leads to $\gamma \simeq 0.51 \pm 0.14$, consistent with the previously reported estimate $(0.60 \pm 0.15)$ and with the expected value $\gamma \approx 0.55$. The error estimate reported in Ref. 10 only took into account this variation, due to dropping various number of points. There is

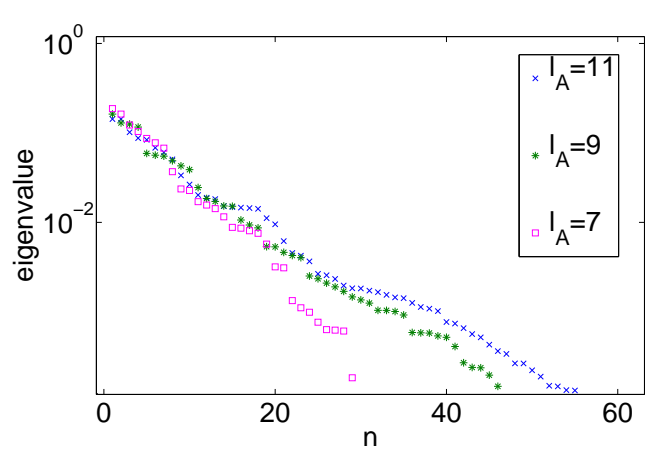

FIG. 7: (Color online.) Density matrix eigenvalues in decreasing order for orbital partitioning with $l_{A}$ orbitals in a block for $N=9, m=3$ Laughlin state.

also some error propagated from the extrapolation uncertainty. Using the conservative uncertainty estimate proposed in the previous subsection gives us a more conservative and more rigorous error estimate, $\gamma \simeq 0.51 \pm 0.25$.

\section{Eigenvalue distribution for reduced density matrix}

In Fig. 7 , we show the largest eigenvalues of reduced density matrices obtained by orbital or spatial partitioning. The eigenvalues are ordered according to decreasing magnitude and plotted on a log scale; the resulting curves are roughly linear, suggesting a roughly exponential decay of the eigenvalue distribution function.

It is interesting to note the complete dissimilarity of this eigenvalue spectrum compared to the particle partitioning case discussed earlier, e.g., Fig. 1. It would also be interesting to put our observations in the context of the spectra of reduced density matrices of many-body systems in general. Reduced density matrices for spatially connected blocks have been studied previously in the context of the convergence of the DMRG algorithm; an overview is available in section III-B of Schollwöck's DMRG review. ${ }^{28}$ From our numeric data, It is difficult to say whether or not the decay of the eigenvalue distributions is slower than exponential.

\section{CONCLUDING REMARKS}

We have presented a detailed study of the entanglement entropy in abelian and non-abelian quantum Hall states, taking a paradigmatic example of each, the $\nu=1 / 3$ Laughlin state and the $\nu=1 / 2$ Moore-Read state.

For entanglement between subsets of particles, we have demonstrated the effects of particle-particle correlations in the deviation of the entanglement entropies from an 
upper bound $S_{A}^{F}$ set by fermionic statistics only. We presented a close upper bounds for particle entanglement entropies for both bosonic and fermionic states. For a particular case $n_{A}=N / 2$, i.e. entanglement of the one half of the system with the other, the presented upper bound is notably lower than a similar estimate in Ref. 17. We showed that the distribution of the eigenvalue spectrum of the two-particle density matrix can be directly related to a two-particle correlation function $g_{2}(r)$. This suggests that the eigenvalue distribution of $n_{A}$-particle reduced density matrices can be related to $n_{A}$-particle correlation functions.

For entanglement between spatial regions, indexed by orbitals on a spherical geometry, we have provided a thorough discussion of our efforts to optimize the procedure for extracting the so-called "topological entropy" from finite-size data. Using this procedure, we calculated the topological entropy for the Moore-Read state, and found our numerical result to be consistent with the expected value $\ln \sqrt{8}$.

Our results on Laughlin and Moore-Read states indicate that the computation of topological entanglement entropies can be used for diagnosing topological order in FQH states that are only available for a limited number of particles. Clearly, the practical use of this method hinges on the accuracy by which a value for $\gamma$ can be extracted. In this context, it could be particularly interesting to use entropies $S_{l_{A}}[N]$ that are generated in DMRG studies of FQH states. ${ }^{29}$

It is interesting to note the differences between the particle and spatial partitioning cases. In the first case, correlation effects decrease the particle entanglement entropy $S_{n_{A}}$. In the latter case, correlation effects increase the spatial entanglement entropy; for example, a Wigner crystal would have very high entanglement entropy between two spatial partitions. These differences are particularly dramatically manifested in the difference between the spectral structure of the reduced density matrices for the two cases. In the particle entanglement case, the eigenvalues tend to be of the same order, with relatively small fluctuations around some average value. In the spatial case, the eigenvalue distribution function has an exponential-like form, and only a small fraction of the eigenvalues contribute to the entanglement entropy.

\section{Acknowledgments}

We thank U. Schollwöck for drawing our attention to the Bulirsh \& Stoer (BST) algorithm for extrapolations and we acknowledge discussions with E. Ardonne (on eq. (19)), P. Calabrese and A. Feiguin. The research of $\mathrm{OZ}$ and $\mathrm{KS}$ is financially supported by the Stichting voor Fundamenteel Onderzoek der Materie (FOM) of the Netherlands. EHR is supported by US DOE under contract DE-FG03-02ER-45981. KS and EHR acknowledge the Institute for Pure and Applied Mathematics (IPAM) at Los Angeles, where part of this work was done.

\section{APPENDIX A: 2-PARTICLE REDUCED DENSITY MATRIX AND CORRELATION FUNCTIONS}

In this appendix we derive Eq. (16). The two-particle reduced density matrix may be written as

$$
\rho_{2}=\sum_{l, m} \lambda_{l m}|l, m\rangle\langle l, m|
$$

where $|l, m\rangle$ is a state of two particles with total angular momentum $l$ and projection $m$. The rotational symmetry of the sphere ( $\rho_{2}$ must be invariant under rotations) indicates that the eigenvalues $\lambda_{l m}$ do not depend on the projection $m$, i.e, are grouped in $S U(2)$ multiplets; $\lambda_{l m}=\lambda_{l}$.

To get the correlation function, we need to compute the density matrix in a basis of polar spherical coordinates,

$$
\rho_{2}\left(\theta_{1}, \theta_{2}, \phi_{1}, \phi_{2}\right)=\left\langle\theta_{1}, \theta_{2}, \phi_{1}, \phi_{2}\left|\rho_{2}\right| \theta_{1}, \theta_{2}, \phi_{1}, \phi_{2}\right\rangle,
$$

where $\theta_{i}$ and $\phi_{i}$ are coordinates of particles on a sphere. Rotational symmetry requires that $\rho_{2}$ depends only on the angular distance $\theta$ between particles:

$$
\cos \theta=\cos \left(\phi_{1}-\phi_{2}\right) \sin \theta_{1} \sin \theta_{2}+\cos \theta_{1} \cos \theta_{2} \text {. }
$$

Now the unnormalized wavefunction of two particles with total angular momentum $l$ can be written $\operatorname{as}^{20}$

$$
\Psi_{l}=\prod_{i=1,2}\left(\alpha^{*} u_{i}+\beta^{*} v_{i}\right)^{l}\left(u_{1} v_{2}-u_{2} v_{1}\right)^{2 L-l}
$$

where $L$ is the angular momentum of each particle, $u_{i}=$ $\cos \frac{\theta_{i}}{2} e^{i \phi_{i} / 2}, v_{i}=\sin \frac{\theta_{i}}{2} e^{-i \phi_{i} / 2}$, and $\alpha, \beta$ fix the center of mass of the two particles. The same wavefunction can also be written as

$$
\Psi_{l}=\left\langle\theta_{1}, \theta_{2}, \phi_{1}, \phi_{2}|\hat{D}(\alpha, \beta)| l, l\right\rangle,
$$

where $\hat{D}(\alpha, \beta)$ is a rotation operator. Let us write $|\Omega\rangle=$ $\hat{D}(\alpha, \beta)|l, l\rangle$, which is a familiar coherent state. Using the identity

$$
\frac{2 l+1}{4 \pi} \int d \Omega|\Omega\rangle\left\langle\Omega\left|=\sum_{m}\right| l, m\right\rangle\langle l, m|
$$

one obtains

$$
\begin{aligned}
& \sum_{m}\left|\left\langle\theta_{1}, \theta_{2}, \phi_{1}, \phi_{2} \mid l, m\right\rangle\right|^{2}= \\
& \frac{2 l+1}{4 \pi} \int d \Omega\left|\left\langle\theta_{1}, \theta_{2}, \phi_{1}, \phi_{2} \mid \Omega\right\rangle\right|^{2} .
\end{aligned}
$$

The normalization of $\left\langle\theta_{1}, \theta_{2}, \phi_{1}, \phi_{2} \mid \Omega\right\rangle$ is equal to ${ }^{30}$

$$
N_{l}=\frac{16 \pi^{2}(l !)^{2}}{(2 l+1) !} \frac{(2 L-l) !(2 L+l+1) !}{(2 L+1) !^{2}} .
$$


We need to compute

$$
\begin{aligned}
I_{l} & \equiv \int d \Omega\left|\left\langle\theta_{1}, \theta_{2}, \phi_{1}, \phi_{2} \mid \Omega\right\rangle\right|^{2} \\
& =\int d \Omega_{\alpha, \beta} \prod_{i=1,2}\left|\alpha^{*} u_{i}+\beta^{*} v_{i}\right|^{2 l}\left|u_{1} v_{2}-u_{2} v_{1}\right|^{4 L-2 l}
\end{aligned}
$$

Defining $a \equiv\left|u_{1} v_{2}-u_{2} v_{1}\right|^{2}=(1-\cos \theta) / 2$ and $b \equiv$ $\left|u_{1}^{*} u_{2}+v_{2} v_{1}^{*}\right|^{2}=(1+\cos \theta) / 2$, one finds

$$
\begin{array}{r}
I_{l}(\theta)=\frac{4 \pi(l !)^{2}}{(2 l+1) !} a^{2 L-l} \sum_{k=0}^{l} \frac{(l+k) !}{(l-k) !(k !)^{2}} a^{l-k} b^{k} \\
\quad=\frac{4 \pi(l !)^{2}}{(2 l+1) !} a^{2 L}{ }_{2} F_{1}(-l, l+1,1,-b / a) .
\end{array}
$$

One can see that $I_{l}$ is normalized as

$$
\int_{0}^{\pi} d \theta \sin \theta I_{l}(\theta)=\frac{N_{l}}{2 \pi} .
$$

Introducing the function

$$
\begin{aligned}
& R_{l}(\theta) \equiv \frac{I_{l}(\theta)}{N_{l}}= \\
& \frac{(2 L+1) !^{2}}{4 \pi(2 L-l) !(2 L+l+1) !} a_{2}^{2 L} F_{1}(-l, l+1,1,-b / a)
\end{aligned}
$$

we finally write the 2-body correlator as

$$
\rho_{2}(\theta)=\sum_{l} \lambda_{l} \frac{2 l+1}{4 \pi} R_{l}(\theta) .
$$

\section{APPENDIX B: ENTANGLEMENT ENTROPIES; NUMERICAL RESULTS WITH ORBITAL PARTITIONING}

In tables II and III we list orbital-partitioning entanglement entropies calculated using the numerical wavefunctions. For each wavefunction (each colmun), the entanglement entropies are only listed up to their maximum value, typeset in bold, because the values after this are determined by the symmetry $S_{l_{A}}=S_{N_{\phi}+1-l_{A}}$. The extrapolation procedure of Sec. IV B involves extrapolating each row of numbers to $N \rightarrow \infty$.

Note that, in each column (for a particular $N$ ), the omitted part after the midpoint (in bold) is a decreasing function of $l_{A}$ and thus does not give useful information about the thermodynamic limit of $S_{l_{A}}$. In the extrapolation, it is therefore important to avoid values from these parts of the table. We therefore restrict ourselves to values which in tables II and III are to the right of (i.e., above) the diagonal line through the midpoint numbers typeset in bold.
1 X. G. Wen and Q. Niu, Phys. Rev. B 41, 9377 (1990); X. G. Wen, Phys. Rev. B 41, 12838 (1990); X. G. Wen, Phys. Rev. B 44, 2664 (1991).

2 G. Moore and N. Read, Nucl. Phys. B360, 362 (1991).

${ }^{3}$ N. Read and E. Rezayi, Phys. Rev. B 59, 8084 (1999).

${ }^{4}$ E. Ardonne and K. Schoutens, Phys. Rev. Lett. 82, 5096 (1999).

5 A. Yu. Kitaev, Annals Phys. 303, 2 (2003); M. Freedman, M. Larsen, and Z. Wang, Commun. Math. Phys. 227, 605 (2002); N. E. Bonesteel, L. Hormozi, G. Zikos, S.H. Simon, Phys. Rev. Lett. 95, 140503 (2005); S. Das Sarma, M. Freedman, C. Nayak, Phys. Rev. Lett. 94, 166802 (2005).

${ }^{6}$ G. Vidal, J. I. Latorre, E. Rico and A. Kitaev, Phys. Rev. Lett. 90, 227902 (2003).

7 J. I. Latorre, E. Rico, and G. Vidal, Quantum Information and Computation, 4, 48 (2004); V.E. Korepin, Phys. Rev. Lett. 92, 096402 (2004); V. Popkov and M. Salerno, Phys. Rev. A 71, 012301 (2005).

8 P. Calabrese and J. Cardy, J. Stat. Mech. 0406, 002 (2004).

9 K. Audenaert, J. Eisert, M. B. Plenio, R. F. Werner, Phys. Rev. A 66, 042327 (2002).

10 M. Haque, O. Zozulya, and K. Schoutens, Phys. Rev. Lett. 98, 060401 (2007).

11 A. Kitaev and J. Preskill, Phys. Rev. Lett. 96, 110404 (2006).
12 M. Levin and X. G. Wen, Phys. Rev. Lett. 96, 110405 (2006).

13 N. Read and E. Rezayi, Phys. Rev. B 54, 16864 (1996).

14 N. Read, Physica 298B, 121 (2001).

15 C. Holzhey, F. Larsen, and F. Wilczek, Nucl. Phys. B 424, 443 (1995).

16 B. Zeng, H. Zhai, and Z. Xu, Phys. Rev. A 66, 042324 (2002).

17 S. Iblisdir, J. I. Latorre, and R. Orús, Phys. Rev. Lett. 98, 060402 (2007).

18 R. Santachiara, F. Stauffer, D. Cabra, cond-mat/0610402; P. Calabrese and M. Mintchev, cond-mat/0703117.

19 S. Furukawa and G. Misguich, cond-mat/0612227.

20 F. D. M. Haldane, Phys. Rev. Lett. 51, 605 (1983).

21 D. P. Arovas, A. Auerbach, and F. D. M. Haldane, Phys. Rev. Lett. 60, 531 (1988).

${ }^{22}$ V. Gurarie and E. Rezayi, Phys. Rev. B 61, 5473 (2000); E. Ardonne, N. Read, E. Rezayi and K.Schoutens, Nucl. Phys. B 607, 549 (2001); E. Ardonne, J. Phys. A 35, 447 (2002).

23 M. Srednicki, Phys. Rev. Lett. 71, 666 (1993); M. M. Wolf, F. Verstraete, M. B. Hastings, J. I. Cirac, arXiv: 0704.3906; M. B. Plenio, J. Eisert, J. Dreißig, M. Cramer, Phys. Rev. Lett. 94, 060503 (2005).

24 P. Fendley, M. P. A. Fisher, and C. Nayak, J. Stat. Phys. 126, 1111 (2007). 


\begin{tabular}{|c||c|c|c|c|c|c|c|c|}
\hline$l_{A}$ & $N=4$ & $N=6$ & $N=8$ & $N=10$ & $N=12$ & $N=14$ & $N=16$ & $N=18$ \\
\hline \hline 1 & 0.636514 & 0.673012 & 0.682908 & 0.686962 & 0.689009 & 0.690186 & 0.690923 & 0.691416 \\
\hline 2 & 1.09861 & 1.15777 & 1.23519 & 1.24961 & 1.25796 & 1.2663 & 1.27095 & 1.27385 \\
\hline 3 & $\mathbf{1 . 0 9 8 6 1}$ & 1.49971 & 1.65513 & 1.7002 & 1.72587 & 1.74591 & 1.75859 & 1.76716 \\
\hline 4 & & 1.76712 & 2.0355 & 2.11077 & 2.15836 & 2.19257 & 2.21444 & 2.22959 \\
\hline 5 & & $\mathbf{1 . 8 8 1 5 2}$ & 2.31807 & 2.44895 & 2.52664 & 2.58123 & 2.61639 & 2.64089 \\
\hline 6 & & & 2.48295 & 2.70298 & 2.82429 & 2.90754 & 2.96113 & 2.99855 \\
\hline 7 & & & $\mathbf{2 . 5 4 2 8 2}$ & 2.88348 & 3.06178 & 3.18053 & 3.257 & 3.31045 \\
\hline 8 & & & & 2.98996 & 3.24156 & 3.40254 & 3.50653 & 3.57927 \\
\hline 9 & & & & $\mathbf{3 . 0 2 5 5 8}$ & 3.36728 & 3.57792 & 3.71402 & 3.80922 \\
\hline 10 & & & & & 3.44165 & 3.71089 & 3.88358 & 4.00428 \\
\hline 11 & & & & & $\mathbf{3 . 4 6 6 0 1}$ & 3.80408 & 4.01821 & 4.16743 \\
\hline 12 & & & & & & 3.85949 & 4.12054 & 4.30135 \\
\hline 13 & & & & & & $\mathbf{3 . 8 7 7 7 4}$ & 4.19219 & 4.408 \\
\hline
\end{tabular}

TABLE II: Orbital-partitioning entanglement entropies $S_{l_{A}}[N]$ for the $\nu=1 / 2$ Moore-Read state.

\begin{tabular}{|c||c|c|c|c|c|c|c|c|}
\hline$l_{A}$ & $N=3$ & $N=4$ & $N=5$ & $N=6$ & $N=7$ & $N=8$ & $N=9$ & $N=10$ \\
\hline \hline 1 & 0.682908 & 0.673012 & 0.666278 & 0.661563 & 0.65811 & 0.655482 & 0.653418 & 0.651757 \\
\hline 2 & 1.00424 & 1.05492 & 1.07339 & 1.0822 & 1.08707 & 1.09006 & 1.09202 & 1.09337 \\
\hline 3 & $\mathbf{1 . 2 7 7 0 3}$ & 1.3944 & 1.44547 & 1.46998 & 1.48491 & 1.49466 & 1.50147 & 1.50648 \\
\hline 4 & $\mathbf{1 . 2 7 7 0 3}$ & 1.59387 & 1.70596 & 1.76262 & 1.79672 & 1.81933 & 1.8353 & 1.84717 \\
\hline 5 & & $\mathbf{1 . 6 6 1 8 4}$ & 1.87354 & 1.97796 & 2.03976 & 2.08068 & 2.10962 & 2.13113 \\
\hline 6 & & & $\mathbf{1 . 9 5 2 3}$ & 2.1202 & 2.21872 & 2.28347 & 2.32919 & 2.36314 \\
\hline 7 & & & $\mathbf{1 . 9 5 2 3}$ & 2.20091 & 2.3448 & 2.43838 & 2.50425 & 2.55307 \\
\hline 8 & & & & $\mathbf{2 . 2 2 7 6 8}$ & 2.42555 & 2.55286 & 2.64196 & 2.70783 \\
\hline 9 & & & & & $\mathbf{2 . 4 6 4 5 1}$ & 2.63139 & 2.74713 & 2.83233 \\
\hline 10 & & & & & $\mathbf{2 . 4 6 4 5 1}$ & 2.67761 & 2.82363 & 2.93049 \\
\hline 11 & & & & & & $\mathbf{2 . 6 9 2 9 3}$ & 2.87356 & 3.00467 \\
\hline 12 & & & & & & & $\mathbf{2 . 8 9 8 1 7}$ & 3.05653 \\
\hline
\end{tabular}

TABLE III: Orbital-partitioning entanglement entropies $S_{l_{A}}[N]$ for the $\nu=1 / 3$ Laughlin state.

25 R. Bulirsh and J. Stoer, Numer. Math. 6, 413 (1964).

${ }^{26}$ M. Henkel and G. Schütz, J. Phys. A 21, 2617 (1988).

27 F.D.M. Haldane, unpublished.

28 U. Schollwöck, Rev. Mod. Phys. 77, 259 (2005).

${ }^{29}$ We thank A. Feiguin for discussions on this and for pro- viding us with DMRG data for large-size Laughlin wavefunctions.

30 G. Fano, F. Ortolani, E. Colombo, Phys. Rev. B 34, 2670, (1986). 